Julie Landour, Sociologie des Mompreneurs. Entreprendre pour concilier travail et famille?

Villeneuve-d'Ascq, Presses universitaires du Septentrion, coll. « Le regard sociologique », 2019

\title{
Gabrielle Schütz
}

\section{(2) OpenEdition}

\section{Journals}

Édition électronique

URL : https://journals.openedition.org/travailemploi/10751

DOI : 10.4000/travailemploi.10751

ISSN : 1775-416X

Éditeur

DARES - Ministère du Travail

Édition imprimée

Date de publication : 15 décembre 2020

Pagination : 144-147

ISSN : 0224-4365

Référence électronique

Gabrielle Schütz, « Julie Landour, Sociologie des Mompreneurs. Entreprendre pour concilier travail et famille ?», Travail et Emploi [En ligne], 163 | 2020, mis en ligne le 01 février 2022, consulté le 26 février 2022. URL : http://journals.openedition.org/travailemploi/10751 ; DOI : https://doi.org/10.4000/ travailemploi. 10751 


\section{Sociologie des Mompreneurs. Entreprendre pour concilier travail et famille?}

Julie Landour

Villeneuve-d'Ascq, Presses universitaires du Septentrion, coll. « Le regard sociologique », 2019, 184 p.

\section{Lu par Gabrielle Schütz*}

Consacré aux «Mompreneurs », ces femmes qui créent leur entreprise après l'arrivée d'un enfant, l'ouvrage tiré de la thèse de Julie Landour éclaire les parcours qui mènent les femmes du salariat à l'indépendance, tout en donnant à voir les normes et pratiques liées à la maternité dans certains espaces sociaux. Il investigue ainsi la thématique de la « conciliation », ou plutôt la manière conflictuelle dont s'articulent et se superposent différents temps sociaux pour les femmes, et, ce faisant, donne à apprécier le renouveau du patriarcat (cf. infra). Il s'appuie principalement sur une enquête ethnographique et statistique réalisée dans l'un des collectifs au sein desquels les Mompreneurs se fédèrent: observations, suivi de ses membres sur Facebook, passation d'un questionnaire $(n=268)$, recueil de récits de vie auprès d'une cinquantaine d'entre elles.

La première partie du livre s'intéresse aux conditions d'émergence des Mompreneurs et dresse leur portrait de groupe. Entré sur la scène médiatique française fin 2008, le mouvement des Mompreneurs s'inscrit dans le renouveau symbolique de l'indépendance et s'adosse aux politiques publiques volontaristes promouvant la création individuelle d'entreprise dans un contexte de chômage élevé. L'analyse du cadre législatif, à travers l'étude de rapports et débats parlementaires, montre que ces politiques n'ont toutefois jamais pensé les femmes comme un public-cible ni fait de la « conciliation » l'un de leurs objectifs. Cette « conciliation » n'est d'ailleurs pas non plus revendiquée par les pionnières qui ont contribué à l'émergence de la catégorie, et ont plutôt mis en avant l'inventivité des mères et leur valeur professionnelle pour tenter d'opérer un retournement du stigmate de la maternité. Majoritairement blanches, issues des classes moyennes et supérieures, hautement qualifiées, les Mompreneurs ont des profils qui relativisent l'idée d'une démocratisation de l'accès à l'indépendance. Alors que leur entrepreneuriat est généralement présenté comme un choix positif, voire une vocation, l'analyse des parcours de ces femmes montre qu'elles étaient souvent en difficulté professionnelle avant de créer leur activité : la maternité leur

* Université de Versailles Saint Quentin, laboratoire Printemps (CNRS/UVSQ). 
ouvre une porte de sortie plutôt qu'elle n'est en tant que telle le véritable déclencheur de leur bifurcation. L'activité qu'elles créent revêt pour beaucoup d'entre elles une dimension identitaire forte, qui leur permet de renouer avec la valeur expressive du travail, souvent absente de leur expérience salariale. Au-delà de ces traits communs, la catégorie est très hétérogène: du point de vue des activités et des statuts juridiques de l'entreprise créée, mais aussi de celui du caractère «accomplissant» ou non de leurs différents engagements. L'autrice propose une typologie qui oppose, sur un premier plan, les Mompreneurs dont l'entreprise est viable à celles pour qui elle fait avant tout office de « façade d'activité » (p. 49). Ces dernières, gagnées à la norme de l'activité féminine, soucieuses de «tenir leur rang professionnel » mais ne tirant que de très faibles revenus de leur activité (59\% des Mompreneurs déclarent un chiffre d'affaires annuel inférieur à 10000 euros), sont qualifiées de « femmes au foyer revisitées par la société de marché » (p. 48). Sur un autre plan, la typologie oppose les Mompreneurs qui s'épanouissent dans leurs activités maternelles aux autres. Finalement, seules les « entrepreneures démultipliées », numériquement minoritaires, incarnent le storytelling sur la catégorie, par leur accomplissement sur tous les fronts. Elles combinent différentes ressources dont toutes les Mompreneurs sont loin de disposer, ressources issues de leur ancienne affiliation salariale (bilan de compétences, formation professionnelle continue, indemnités chômage, réseau professionnel facilitant la constitution d'une clientèle, etc.) et/ou d'un habitus entrepreneurial.

Dans une deuxième partie, l'autrice spécifie l'engagement maternel des Mompreneurs. Investies dans un projet parental exigeant et « puérocentré » (p. 85 et suivantes), qu'elles présentent comme partagé avec leur conjoint, elles sont pour la moitié d'entre elles le principal mode de garde de leur enfant. Au-delà de la prise en charge matérielle du ou des enfants, il s'agit de garantir leur sécurité affective et leur bien-être psychologique grâce à une disponibilité extensive, conjuguée exclusivement au féminin. L'autrice décrit le poids concret de la maternité sur l'engagement professionnel salarié (discriminations, burn-out), tout en documentant les répercussions professionnelles des parcours d'adoption ou de procréation médicalement assistée (PMA). Elle donne à voir l'incompatibilité des injonctions portées par les « entrepreneurs de la morale familiale ${ }^{1} »($ p. 94) d'une part, et par les organisations salariales d'autre part. Toutefois, elle analyse également la maternité sous l'angle des profits de distinction qu'elle procure, en introduisant le concept de « capital parental » (p. 111 et suivantes) comme une variante du capital culturel. Pour une partie des Mompreneurs, dont l'activité est directement liée au «marché de l'enfance » (confection de vêtements de grossesse, coaching parental, etc.), le capital parental se traduit par une expertise qu'elles peuvent vendre avec succès si elles disposent par ailleurs des ressources adéquates pour leur projet entrepreneurial. Pour les autres, le capital parental fait office de «marchepied efficace pour se positionner dans les hiérarchies parentales contemporaines » (p. 116). Il leur permet de se valoriser et d'apparaître comme de

1. L'autrice fait référence à l'article de Muriel Darmon (1999), « Les “entreprises” de la morale familiale », French Politics, Culture \& Society, vol. 17, n 3-4, pp. 1-19. 
«bonnes » mères dans les relations avec les professionnels de la petite enfance ou avec les autres parents, voire d'opérer une réhabilitation identitaire dans un contexte de difficultés professionnelles.

La dernière partie de l'ouvrage solde les comptes de la double entreprise des Mompreneurs, professionnelle et parentale. À rebours des versions idéalisées qu'elles livrent sur la «conciliation », l'enquête révèle l'emprise du domestique dans leur quotidien, y compris chez celles dont les entreprises sont les plus florissantes: emprise spatiale (79\% travaillent à domicile, $31 \%$ sans espace dédié, voyant leur travail subordonné aux rythmes familiaux) et emprise temporelle (moins de la moitié déclarent consacrer à leur activité l'équivalent d'un temps plein). La « liberté » de s'organiser associée à l'indépendance est " paradoxalement contreproductive pour les femmes » (p. 131), ce qui ne manque pas d'interpeller à l'heure du développement sans précédent du télétravail. Elle conduit les Mompreneurs dans un piège où faible rentabilité de leur activité et assignation domestique s'entretiennent mutuellement. Ce cercle vicieux profite néanmoins à leurs conjoints : bénéficiant de leur travail domestique gratuit, ils économisent des frais de garde et de ménage tout en dégageant des marges temporelles pour davantage développer leurs propres activités professionnelles. Mal à l'aise dans les habits de la femme au foyer sous dépendance conjugale, certaines mettent à contribution leur épargne pour continuer à participer aux dépenses du ménage, ou payer seules un mode de garde de leur enfant qui finit par faire figure de dépense somptuaire. Les séparations conjugales font éclater au grand jour les fissures de la «façade d'activité »: si « la classe limite la casse » (p. 148) pour ces femmes de milieux favorisés, le genre joue à plein et la rupture se solde par un double déclassement, symbolique avec un retour au salariat par l'exercice de métiers à dimension moins expressive, et économique. L'autrice conclut que les Mompreneurs fournissent « une version actualisée et néo-libérale du patriarcat, qui est d' autant plus gênante qu'elle est masquée par un dispositif public vanté pour son égalitarisme et sa liberté » (p. 165).

Très abouti dans sa conceptualisation (de nombreux encadrés précisent avec rigueur les notions utilisées), moins disert sur la méthodologie (on en sait peu par exemple sur le cheminement de l'enquête et la manière dont elle a été négociée avec le collectif de Mompreneurs, ou sur les modalités de construction de la typologie), le livre de J. Landour est extrêmement intéressant. En faisant fructueusement dialoguer sociologie de l'emploi indépendant, de la famille et du genre, il apporte une triple contribution. Il renouvelle la sociologie critique de l'auto-entrepreneuriat (la moitié des Mompreneurs ont créé leur entreprise sous ce statut) et de ses fausses promesses d'émancipation, en dévoilant comment ce régime produit une indépendance en trompel'œil, non pas parce que les Mompreneurs seraient des salariées déguisées, mais parce que leur indépendance se fait sous dépendance conjugale. L'ouvrage documente ainsi l'indépendance « au féminin » en montrant qu'elle ne fait pas l'objet d'un projet de couple, et que la mobilisation conjugale n'est ici pas au rendez-vous, alors qu'elle contribue au succès des indépendants hommes en apportant à leur entreprise une 
contribution décisive bien qu'invisibilisée ${ }^{2}$. On pourra regretter qu'il dialogue moins avec la sociologie du travail et de l'activité: ne peut-on envisager, au-delà de leur trajectoire et des capitaux dont elles disposent, que le secteur économique dans lequel les Mompreneurs s'inscrivent et les caractéristiques de leur activité ont aussi un impact sur leur capacité à créer un auto-emploi viable plutôt qu'une «façade d'activité », et qu'ils induisent des modalités distinctes d'articulation des temps professionnels et domestiques? Ensuite, le livre propose une perspective très stimulante en montrant que la maternité intensive ne constitue pas un refuge pour les seules femmes de milieu populaire et constitue un front sur lequel se jouent des stratégies de distinction. On peut toutefois émettre quelques réserves sur la notion de «capital parental». Si les pratiques des Mompreneurs sont distinctives dans certaines régions de l'espace social, on peut s'interroger sur l'étendue de leur légitimité et de leur efficience en dehors de ces espaces et en dehors du temps circonscrit de la petite enfance. Autrement dit, si les pratiques maternelles ont un potentiel classant, il est moins certain qu'elles confèrent un réel pouvoir, et tout l'ouvrage démontre l'hasardeuse convertibilité de ce "capital parental » en d'autres formes de capital, économique en particulier. Enfin, le livre dissèque avec brio la transformation des modes de domination et les nouveaux atours de l'oppression féminine. Malgré une dénomination oxymorique alliant deux figures éminemment genrées, la mère et l'entrepreneur, les Mompreneurs ne parviennent ni à troubler le genre ni à «prendre la "conciliation" à son propre jeu» (p. 16). La délégitimation des femmes au foyer, la norme de l'activité féminine et la norme de l'égalité, combinées à l'idéologie néo-libérale de l'entreprise de soi, les piègent peutêtre plus sûrement encore que le patriarcat « classique », puisqu'il leur faut à tout prix « donner le change ». Quitte à s'appauvrir en maintenant à flot des entreprises parfois déficitaires ou en contribuant aux frais du ménage au-delà de leur capacité financière, dans un déni de leur dépendance et, plus généralement, des inégalités de genre du monde professionnel.

2. Bertaux-Wiame I. (2004), « Devenir indépendant, une affaire de couple », Cahiers du genre, $\mathrm{n}^{\circ} 37$, pp. 13-40; Samak M. (2017), « Le prix du “retour" chez les agriculteurs “néo-ruraux". Travail en couple et travail invisible des femmes », Travail et Emploi, ${ }^{\circ}$ 150, pp. 53-78; Bajard F. (2020), « De l'atelier à la cuisine chez les céramistes: arrangements de couple et inégalités de genre dans un métier indépendant "égalitariste” », Travail et Emploi, n” 161, pp. 61-91. 